\title{
A Tetra-State Hopping Robot Driven by Shape Memory Alloy Spring Tianqi Liu ${ }^{1, a}$, Shuping Chen ${ }^{1, b}$, Jianglong Guo ${ }^{1}$, Zhilong Dou ${ }^{1}$, Chun Liu ${ }^{1}$,
Qingchun Zhang
}

${ }^{1}$ School of Mechanical Engineering and Automation, Northeastern University, Shenyang 110819, China

aliutianqixin@163.com , bspchen@mail.neu.edu.cn

Keywords: SMA, Hopping robot, Tetra-state cycle, SMA spring

Abstract. According to the characteristics of jumping locomotion, a solution of Tetra-state Hopping Robot (for short: THR) actuated by Shape Memory Alloy Spring (for short: SMAS) is presented in this paper. This article establishes a mechanical model of the jumping robot and analyzes the kinematics and the dynamics of the mechanical model. Lagrange method is applied to analyze the dynamics the mechanical model. Meanwhile, a preliminary explanation of the THR flipping phenomenon during the take-off and flight process is conducted from the perspective of momentum moment.

\section{Hopping Robot with a Tetra-state Cycle}

We have successfully developed a THR, as shown in Fig. 1, which has a tetra-state cycle and is actuated by SMAS. The THR has great differences with many prototype machines developed by domestic and foreign institutions ${ }^{[1]}$. The tetra-state of the THR: resetting, energy storing, triggering and hopping are all driven by SMAS. It has been validated that the THR can assist Micro Flapping-wing Air Vehicle (for short: MFAV) to take-off, as shown in Fig. 2. The experiment method and equipment will be issued in other articles. Total weight of the THR, including its control system, is $11.1 \mathrm{~g}$ and total height is $55 \mathrm{~mm}$. The THR can jump $480 \mathrm{~mm}$ high and $520 \mathrm{~mm}$ in horizontal distance.

This article analyzes the kinematics and the dynamics of the mechanical model and preliminarily explicates the THR flipping phenomenon during the take-off and flight process from the perspective of momentum moment.

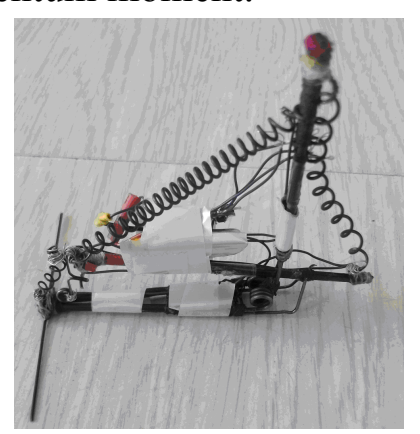

Fig. 1. Jumping prototype with the control system

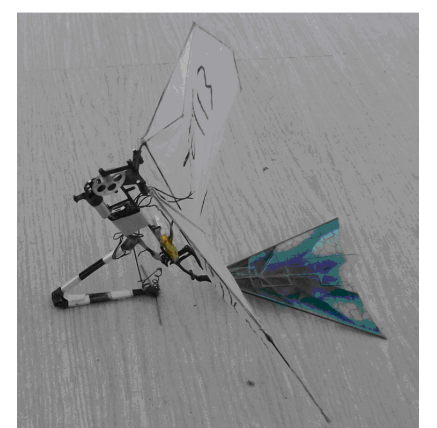

Fig. 2. FMAV with the mechanism of jumping

\section{Mechanical Model of THR}

THR in this article is light and small, and can recover to original state for next bouncing after a take-off. As shown in Fig. 3, the THR is comprised of resetting SMAS, differential SMAS, trigger mechanism, torsional spring, rotation mechanism, adjustment pillar, former arm and back arm.

The work principle of the THR is as follows: under the control of control system, the resetting SMAS 8 shrinks for being heated. Then, the rotation mechanism rotates and stretches the differential SMAS 6 downward. The first hooker 4 at this end will contact the second hooker 3 on one end of the SMAS trigger spring 2 and get locked with it. Meanwhile, some energy of heating the differential SMAS 6 is transformed and restored in the second torsional spring 10. Afterwards, the trigger SMAS 
2 shrinks to unlock the first hooker 4 and the second hooker 3. As a result, the energy restored in the second torsional spring 10 releases and the THR starts to jump. At the same time, the resetting SMAS 8 resets, and if being heated again, the next work loop will begin.

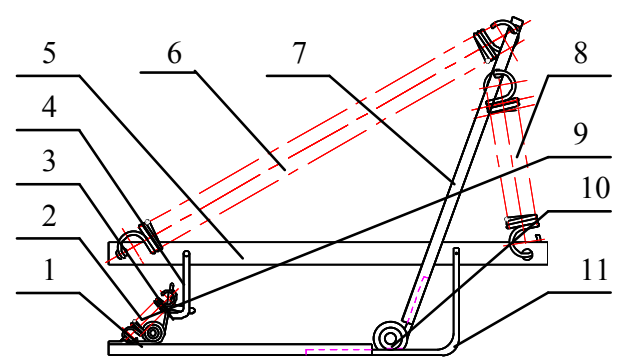

1. back arm 2. trigger mechanism 3.second hooker 4. first hooker 5. rotating mechanism 6. differential SMAS 7. former arm 8. resetting SMAS 9. first torsional spring 10. second torsional spring 11. adjustment pillar.

Fig. 3. Principle diagram of THR.

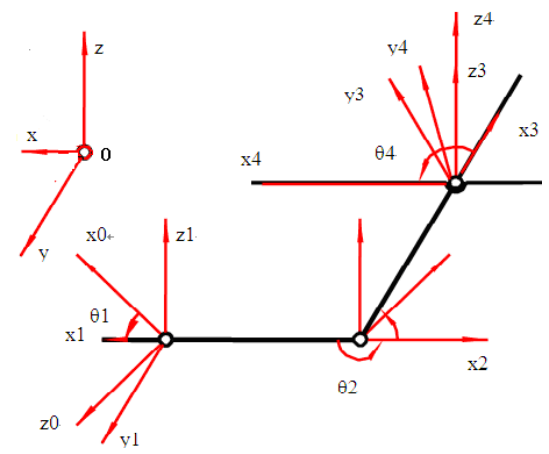

Fig. 4. Scheme of THR

\section{Kinematical Analysis of THR}

Table 1 Variables of structural parameters in THR take-off process

\begin{tabular}{ccccc}
\hline $\begin{array}{c}\text { Mark of part } \\
\text { i }\end{array}$ & $\begin{array}{c}\text { Setover of } \\
\text { joint } \\
/ \mathrm{mm}\end{array}$ & $\begin{array}{c}\text { Rotating angles } \\
\text { of jumping } \\
\theta_{\mathrm{i} / \mathrm{deg}}\end{array}$ & $\begin{array}{r}\text { Length } a_{j} \\
/ \mathrm{mm}\end{array}$ & $\begin{array}{c}\text { Torsional angle } \\
\text { of joint } \\
\alpha_{i j} / \mathrm{deg}\end{array}$ \\
\hline 1 & 0 & $\theta_{1}$ & 11 & 0 \\
2 & 0 & $\theta_{2}$ & 12 & 0 \\
3 & 0 & $\theta_{3}$ & 13 & 0 \\
4 & 0 & $\theta_{4}$ & 14 & 0 \\
\hline
\end{tabular}

According to D-H coordinate method, the conjoined coordinate system of the mechanical model is established and shown in Fig. 4. The jumping process of the mechanical model can be separated into take-off process and flight process. As shown in table 1, variables of structural parameters in take-off process of the mechanical model have been ascertained.

Pose Matrix of Centroid Kinematic Parameters for Take-off. Homogeneous transformation matrixes of each component of the conjoined coordinate system $\{i\}$ are as follows:

$$
{ }_{1}^{0} T=\left[\begin{array}{cccc}
\mathrm{c} \theta_{1} & 0 & \mathrm{~s} \theta_{1} & l_{1} c \theta_{1} \\
-s \theta_{1} & 0 & c \theta_{1} & l_{1} \mathrm{~s} \theta_{1} \\
0 & 1 & 0 & 0 \\
0 & 0 & 0 & 1
\end{array}\right],{ }_{2}^{1} T=\left[\begin{array}{cccc}
-1 & 0 & 0 & -l_{2} c \theta_{2} \\
-s \theta_{2} & -1 & 0 & l_{2} s \theta_{2} \\
0 & 0 & 1 & 0 \\
0 & 0 & 0 & 1
\end{array}\right], \quad{ }_{3}^{2} T=\left[\begin{array}{cccc}
\mathrm{c} \theta_{3} & s \theta_{3} & 0 & l_{3} c \theta_{3} \\
-s \theta_{3} & c \theta_{3} & 0 & l_{3} s \theta_{3} \\
0 & 0 & 1 & 0 \\
0 & 0 & 0 & 1
\end{array}\right],{ }_{4}^{3} T=\left[\begin{array}{cccc}
\mathrm{c} \theta_{4} & s \theta_{4} & 0 & -l_{4} \mathrm{c} \theta_{4} \\
-s \theta_{4} & c \theta_{4} & 0 & l_{4} \mathrm{~s} \theta_{4} \\
0 & 0 & 1 & 0 \\
0 & 0 & 0 & 1
\end{array}\right] .
$$

The centroid of the mechanical model can be calculated as the following Eq. (1).

$$
\left\{\begin{array}{l}
\mathrm{a}=\frac{\sum_{i=1}^{4} m_{i} x_{i}}{\sum_{i=1}^{4} m_{i}}=\frac{m_{1} l_{1} c \theta_{1}-m_{2} l_{2} c \theta_{2}+m_{3} l_{3} c \theta_{3}-m_{4} l_{4} c \theta_{4}}{m_{1}+m_{2}+m_{3}+m_{4}} \\
b=\frac{\sum_{i=1}^{4} m_{i} y_{i}}{\sum_{i=1}^{4} m_{i}}=\frac{m_{1} l_{1} s \theta_{1}+m_{2} l_{2} s \theta_{2}+m_{3} l_{3} s \theta_{3}+m_{4} l_{4} s \theta_{4}}{m_{1}+m_{2}+m_{3}+m_{4}} \\
c=0
\end{array}\right.
$$


Homogeneous transformation matrix of the centroid relating to the fourth perch is as follows:

$$
{ }_{S}^{4} T=\left[\begin{array}{llll}
1 & 0 & 0 & a \\
0 & 1 & 0 & b \\
0 & 0 & 1 & c \\
0 & 0 & 0 & 1
\end{array}\right] .
$$

Then, total transformation matrix of the centroid relating to the fixed reference coordinate $\{\mathrm{O}\}$ can be solved in Eq. (2).

$$
\begin{aligned}
& { }_{S}^{0} T={ }_{1}^{0} T_{2}^{1} T_{3}^{2} T_{4}^{3} T_{S}^{4} T \\
& =\left[\begin{array}{cccc}
-\mathrm{c} \theta_{1} \mathrm{c} \theta_{34} & -c \theta_{1} s \theta_{34} & \mathrm{~s} \theta_{1} & -c \theta_{1}\left(a c \theta_{34}+b s \theta_{34}-l_{4} c \theta_{34}+l_{3} c \theta_{3}+l_{2} \mathrm{c} \theta_{2}-l_{1}\right) \\
s \theta_{1} \mathrm{c} \theta_{34} & s \theta_{1} s \theta_{34} & c \theta_{1} & s \theta_{1}\left(a c \theta_{34}+b s \theta_{34}-l_{4} c \theta_{34}+l_{3} c \theta_{3}-l_{2} c \theta_{2}+l_{1}\right) \\
s \theta_{34}-s \theta_{2} c \theta_{34} & -s \theta_{2} s \theta_{34}-c \theta_{34} & 0 & a\left(s \theta_{34}+s \theta_{2} c \theta_{34}\right)+b\left(-s \theta_{2} s \theta_{34}-c \theta_{34}\right)-l_{4} s \theta_{34}+l_{4} s \theta_{2} c \theta_{34}-l_{3} s \theta_{2} c \theta_{3}-l_{3} s \theta_{3}+l_{2} s \theta_{2} \\
0 & 0 & 0 & 1
\end{array}\right] .
\end{aligned}
$$

The position of the centroid in the fixed-coordinate $\{\mathrm{O}\}$ is symbolized as $\left(\mathrm{x}_{\mathrm{s}}, \mathrm{y}_{\mathrm{s}}, \mathrm{z}_{\mathrm{s}}\right)$. If the rotating angle of the four joints and the length of the four poles are known, the position of the centroid can be obtained by Eq. (3).

$$
\left\{\begin{array}{l}
x_{S^{\prime}}=-c \theta_{1}\left(a c \theta_{34}+b s \theta_{34}-l_{4} c \theta_{34}+l_{3} c \theta_{3}+l_{2} c \theta_{2}-l_{1}\right) \\
y_{S^{\prime}}=s \theta_{1}\left(a c \theta_{34}+b s \theta_{34}-l_{4} c \theta_{34}+l_{3} c \theta_{3}-l_{2} c \theta_{2}+l_{1}\right) \\
z_{S^{\prime}}=a\left(s \theta_{34}+s \theta_{2} c \theta_{34}\right)+b\left(-s \theta_{2} s \theta_{34}-c \theta_{34}\right)-l_{4} s \theta_{34}+l_{4} s \theta_{2} c \theta_{34}-l_{3} s \theta_{2} c \theta_{3}-l_{3} s \theta_{3}+l_{2} s \theta_{2}
\end{array}\right.
$$

The linear velocity and the angular velocity of the centroid about the fixed reference coordinate $\{\mathrm{O}\}$ can be separately expressed in Jacobin matrix form:

$$
\mathrm{v}=J_{\mathrm{cv}} \dot{q}_{1}, w=J_{c w} \dot{q}_{1} .
$$

where, $\dot{q}_{1}=\left[\begin{array}{lll}\dot{\theta}_{1} & \dot{\theta}_{2} & \dot{\theta}_{3}\end{array}\right]$ symbolizes the generalized velocity vector of each joint; $J_{c v}$ represents the linear velocity Jacobin matrix of the body mass center.

Apparently, the angular acceleration vector of the centroid is the derivative form of Eq. (4), and it is shown in Eq. (5).

$$
\left\{\begin{array}{c}
\dot{v}=J_{c v} \ddot{q}_{1}+\dot{J}_{c v} \dot{q}_{1} \\
\dot{w}=J_{c w} \ddot{q}_{1}+\dot{J}_{c w} \dot{q}_{1}
\end{array}\right.
$$

Kinematics Analysis in Flight Process. Flight process is a stage that THR does not contact with ground. Movement in the flight process can be seen as projectile motion affected merely by gravity. And the projectile motion can be divided into a uniform linear motion in horizontal direction and an upright parabolic motion in vertical direction. Air resistance is neglected. The projecting velocity is assumed as $v_{0}$ and $\alpha, \beta, \gamma$ are angles between $v_{0}$ and axis $\mathrm{x}, \mathrm{y}, \mathrm{z} . g$ symbolizes acceleration of gravity. The projection of the projectile motion on XZ plane of the fixed reference coordinate $\{\mathrm{O}\}$ is shown in Fig. 5. In an instantaneous moment $t$, the displacement, velocity and acceleration of the centroid in direction of axis $\mathrm{x}_{1}, \mathrm{y}_{1}, \mathrm{z}_{1}$ can be obtained as follows:

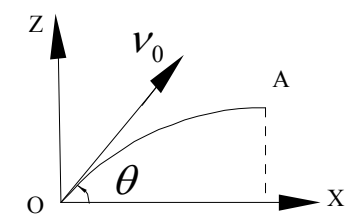

Fig. 5. Projectile motion along XZ 


$$
\left\{\begin{array}{l}
x_{1}=v_{0} \cdot \cos \alpha \cdot t \\
y_{1}=v_{0} \cdot \cos \beta \cdot t \\
z_{1}=v_{0} \cdot \cos \gamma \cdot t-\frac{1}{2} g t^{2}
\end{array},\left\{\begin{array}{c}
v_{x 1}=v_{0} \cdot \cos \alpha \\
v_{y 1}=v_{0} \cos \beta \\
v_{z 1}=v_{0} \cos \gamma-g t
\end{array},\left\{\begin{array}{c}
a_{x 1}=0 \\
a_{y 1}=0 \\
a_{z 1}=-g
\end{array}\right.\right.\right.
$$

where, $v_{0}=\sqrt{v_{x 1}^{2}+v_{y 1}^{2}+v_{z 1}^{2}}, \cos \alpha=\frac{v_{x 1}}{v_{0}}, \cos \beta=\frac{v_{y 1}}{v_{0}}, \cos \gamma=\frac{v_{z 1}}{v_{0}}$.

The maximum height and the biggest leap distance of body mass center in flight process can be acquired by solving Eq. (6) and are separately expressed in Eq. (7) and Eq. (8).

$$
\begin{aligned}
& \mathrm{h}=v_{0} \cos \gamma \cdot \frac{v_{0} \cos \gamma}{g}-\frac{1}{2} g\left(\frac{v_{0} \cos \gamma}{g}\right)^{2}=\frac{v_{0}^{2} \cos ^{2} \gamma}{2 g} . \\
& S=\sqrt{s_{x 1}^{2}+s_{y 1}^{2}}=\sqrt{\frac{4 v_{0}^{4}\left(\cos ^{2} \alpha+\cos ^{2} \gamma\right)}{g^{2}}} \cos \beta=\frac{2 v_{0}^{2} \sqrt{\left(\cos ^{2} \alpha+\cos ^{2} \gamma\right)}}{g} \cos \beta
\end{aligned}
$$

\section{Dynamic Analysis of THR}

The model of the THR can be regarded as bar mechanism of single open-chain or single closed chain. Therefore, it can also be seen as the manipulator's dynamic problem. As the Lagrange method can solve those complex systems' dynamic equations in the simplest form, so dynamics model of this article can be analyzed by using Lagrange principle. This article established the bar's specific geometry and inertia parameters that express the jumping robot's equations of motion of each joint.

Equations in Take-off Process. Seeing the mechanism model as a simple system of mass points, in which masses and velocities of each point are $m_{i}$ and $v_{i}$. According to kinetic energy theorem of particles system, differential form of the particles kinetic energy is as follows:

$$
d K_{i}=d\left(\frac{1}{2} m_{i} v_{i}^{2}\right) \text {. }
$$

Supposing the position of an arbitrary point $P_{i}$ in each corresponding pole is as follows:

$$
{ }^{0} r_{i}=T_{i}{ }^{i} r \text {. }
$$

where, ${ }^{0} r_{i}$ symbolizes the position vector of point ' $P_{i}$ ' is in the fixed reference coordinate system $\{\mathrm{O}\}$, ${ }^{i} r$ stands for the position vector of point ' $P_{i}$ ' in the conjoined coordinate system $\{i\}$, and $T_{i}$ represents the homogeneous transformation matrix from the conjoined coordinate system to the fixed coordinate system reference coordinate $\{\mathrm{O}\}$.

The speed of point ' $P_{i}^{\prime}$ ', Eq. (11), is acquired from the derivative of Eq. (10).

$$
v=\frac{d}{d t}\left({ }^{0} r_{i}\right)=\frac{d}{d t}\left(T_{i}{ }^{i} r\right)=\dot{T}_{i}{ }^{i} r
$$

After $i$ times of transformation, the velocity of point ' $P_{i}$ ' in connecting rod $i$ will be Eq. (12). The trace of matrix, expressed in Eq. (13), can be got by squaring $v$ in order to obtain the kinetic energy of the mechanical model.

$$
\begin{aligned}
& v=\left(\sum_{j=1}^{i} \frac{\partial T_{i}}{\partial \theta_{j}} \dot{\theta}_{j}\right){ }^{i} r . \\
& v^{2}=v v=\operatorname{Trace}\left(v v^{T}\right)=\operatorname{Trace}\left[\sum_{j=1}^{i} \sum_{k=1}^{i} \frac{\partial T_{i}}{\partial \theta_{j}} \cdot{ }^{i} r \cdot\left({ }^{i} r\right)^{T} \cdot\left(\frac{\partial T_{i}}{\partial \theta_{k}}\right)^{T} \dot{\theta}_{j} \dot{\theta}_{k}\right] .
\end{aligned}
$$

The kinetic energy of connecting rod $i$ can be acquired by substituting Eq. (13) into Eq. (9), as shown in Eq. (14).

$$
K_{i}=\int d K_{i}=\frac{1}{2} \operatorname{Trace}\left[\sum_{j=1}^{i} \sum_{k=1}^{i} \frac{\partial T_{i}}{\partial \theta_{j}}\left(\int{ }^{i} r \cdot{ }^{i} r^{T} d m_{i}\right)\left(\frac{\partial T_{i}}{\partial \theta_{k}}\right)^{T} \dot{\theta}_{j} \dot{\theta}_{k}\right] .
$$


Potential Energy of System. Select the ground as zero potential surfaces. Gravitational potential energy of the particle $Q$ which locates ir of connecting rod $i$ is as follows:

$$
P=-m g \cdot \vec{r} \text {. }
$$

Suppose the centroid position of connecting rod $i$ can be expressed by vector ${ }^{i} \vec{r}_{i}$, so potential energy relating to coordinate $T_{i}$ can be obtained as shown in Eq. (15).

$$
P_{i}=-m_{i} g^{T} T_{i}{ }^{i} \vec{r}_{i}
$$

Sequentially, total potential energy will be got and is shown in Eq. (16).

$$
P_{i}=\int_{i} \mathrm{~d} P_{i}=\int_{\mathrm{i}} g^{T} T_{i}{ }^{i} \vec{r}_{i} d m_{i}=g^{T} T_{i} \int_{\mathrm{i}}{ }^{i} \vec{r}_{i} d m_{i}=m_{i} g^{T} T_{i}{ }^{i} r_{i} \text {. }
$$

Former arm and back arm can be regard as homogeneous perch. The centroid position of each perch relating to its conjoined coordinate system $\{\mathrm{i}\}$ is as follow:

${ }^{1} r_{1}=\left[\begin{array}{llll}-\frac{1}{2} l_{1} & 0 & 0 & 1\end{array}\right]^{T},{ }^{2} r_{2}=\left[\begin{array}{llll}-\frac{1}{2} l_{2} & 0 & 0 & 1\end{array}\right]^{T},{ }^{3} r_{3}=\left[\begin{array}{llll}\frac{1}{2} l_{3} & 0 & 0 & 1\end{array}\right]^{T},{ }^{4} r_{4}=\left[\begin{array}{llll}-\frac{1}{2} l_{4} & 0 & 0 & 1\end{array}\right]^{T}$.

Consequently, total potential energy of single arm is shown in Eq. (17).

$$
P=\sum_{i=1}^{4} m_{1} g^{T} T_{1}{ }^{1} r_{1}+m_{2} g^{T} T_{2}{ }^{2} r_{2}+m_{3} g^{T} T_{3}{ }^{3} r_{3}+m_{4} g^{T} T_{4}{ }^{4} r_{4} \text {. }
$$

According to Lagrange method, Lagrange operator can be calculated by Eq. (18).

$$
L=K-P=\frac{1}{2} \sum_{i=1}^{4} \sum_{j=1}^{i} \sum_{k=1}^{i} \operatorname{Trace}\left[\frac{\partial T_{i}}{\partial \theta_{j}} I_{i}\left(\frac{\partial T_{i}}{\partial \theta_{k}}\right)^{T}\right] \dot{\theta}_{\mathrm{j}} \dot{\theta}_{k}-\sum_{i=1}^{4} m_{i} g^{T} T_{i}{ }^{i} r_{i} .
$$

Kinetic equation in take-off process is acquired by the derivation of the Lagrange function (Eq. (18)) and is expressed in Eq. (19).

$$
\begin{aligned}
& F_{i}=\frac{d}{d t}\left(\frac{\partial L}{\partial \dot{\theta}_{p}}\right)-\frac{\partial L}{\partial \theta_{p}} \\
& =\sum_{i=p}^{4} \sum_{k=1}^{i} \operatorname{Trace}\left(\frac{\partial T_{i}}{\partial \theta_{k}} I_{i} \frac{\partial T_{i}^{T}}{\partial \theta_{p}}\right) \ddot{\theta}_{k}+\sum_{i=p}^{4} \sum_{k=1}^{i} \sum_{m=1}^{i} \operatorname{Trace}\left(\frac{\partial^{2} T_{i}}{\partial \theta_{k} \partial \theta_{m}} \cdot I_{i} \cdot \frac{\partial T_{i}^{T}}{\partial \theta_{p}}\right) \cdot \dot{\theta}_{k} \dot{\theta}_{m}-\sum_{i=p}^{4} m_{i} g^{T} \cdot \frac{\partial T_{i}}{\partial \theta_{p}} \cdot{ }^{i} \vec{r}_{i} . \\
& =\sum_{k=1}^{4} D_{i j} \ddot{\theta}_{k}+\sum_{i=1}^{4} \sum_{k=1}^{4} D_{i k m} \dot{\theta}_{m} \dot{\theta}_{k}+D_{i}
\end{aligned}
$$

Generalized coordinates vector, generalized speed and acceleration vector of all joints for THR will be respectively got, as shown in Eq. (20).

$$
q_{1}=\left[\begin{array}{llll}
\theta_{1} & \theta_{2} & \theta_{3} & \theta_{4}
\end{array}\right]^{T} ; \quad \dot{q}_{1}=\left[\begin{array}{llll}
\dot{\theta}_{1} & \dot{\theta}_{2} & \dot{\theta}_{3} & \dot{\theta}_{4}
\end{array}\right]^{T} ; \quad \ddot{q}_{1}=\left[\begin{array}{llll}
\ddot{\theta}_{1} & \ddot{\theta}_{2} & \ddot{\theta}_{3} & \ddot{\theta}_{4}
\end{array}\right]^{T} .
$$

As a result, kinetic equation of THR can be written as Eq. (21).

$$
D\left(q_{1}\right) \ddot{q}_{1}+P\left(q_{1}, \dot{q}_{1}\right)=M .
$$

where,

$$
D\left(q_{1}\right)=\left[\begin{array}{cccc}
D_{11} & D_{12} & D_{13} & D_{14} \\
D_{21} & D_{22} & D_{23} & D_{24} \\
D_{31} & D_{32} & D_{33} & D_{34} \\
D_{41} & D_{42} & D_{43} & D_{44}
\end{array}\right], P\left(q_{1}, \dot{q}_{1}\right)=\left[\begin{array}{l}
\dot{q}_{1}^{T} D^{1} \dot{q}_{1}+D_{1} \\
\dot{q}_{1}^{T} D^{2} \dot{q}_{1}+D_{2} \\
\dot{q}_{1}^{T} D^{3} \dot{q}_{1}+D_{3} \\
\dot{q}_{1}^{T} D^{4} \dot{q}_{1}+D_{4}
\end{array}\right], \quad D^{i}=\left[\begin{array}{cccc}
D_{i 11} & D_{i 12} & D_{i 13} & D_{i 14} \\
D_{i 21} & D_{i 22} & D_{i 23} & D_{i 24} \\
D_{i 31} & D_{i 32} & D_{i 33} & D_{i 34} \\
D_{i 41} & D_{i 42} & D_{i 43} & D_{i 44}
\end{array}\right], i=1,2,3,4
$$

In Eq. (21), joint driving moment vector $M$ can be described as the following. $M=\left[\begin{array}{llll}M_{1} & M_{2} & M_{3} & M_{4}\end{array}\right]^{T}$.

\section{Momentum Moment Analysis in Take-off and Flight Process}

The rotation of the THR can also be explained in momentum moment perspective. In ground phase defined as a stage before the THR taking off, control system heats the differential SMAS 6 to store energy in the second torsional spring 10. As a result, every part of the THR will generate a momentum 
moment to the centroid of THR, and the sum of those momentum moments is not null, because two forces exist at the ground phase. One is support force $N$ given by ground and the other is gravity $G$ of the THR. According to momentum moment of particles system, the time derivative of THR momentum moment to the centroid $Q$ equals the momentum moment of external forces to the same point and Eq. (22) will be acquired. Additionally, the momentum moment of gravity $G$ to the centroid is zero, so Eq. (23) will be obtained.

$$
\frac{\mathrm{d}}{d t} M_{Q}\left(m_{i} v_{i}\right)=M_{Q}(G)+M_{Q}(N) \text {. }
$$

Simplify Eq. (22) and Eq. (23) will be got.

$$
\frac{\mathrm{d}}{d t} M_{Q}\left(m_{i} v_{i}\right)=M_{Q}(N) \text {. }
$$

In the flight process, $N=0$. According to the momentum moment theorem of the particles system, Eq. (24) will be obtained.

$$
\frac{\mathrm{d} M_{Q}\left(m_{i} v_{i}\right)}{d t}=M_{Q}(G)=0
$$

Integrating Eq. (24), Eq. (25) appears.

$$
M_{Q}\left(m_{i} v_{i}\right)=J w=c .
$$

where, $c$ is a constant.

According to the momentum conservation theorem of the particles system, the momentum moment of centroid $Q$ will be obtained as the following.

$$
M_{Q}\left(m_{i} v_{i}\right)=c=\int_{\mathrm{t}_{0}}^{t_{1}} M_{Q}(N)=\int_{0}^{t_{1}}\left(\sum_{i=1}^{4} m_{i} a_{s x} L_{s z}+\sum_{i=1}^{4} m_{i} a_{s z} L_{s x}+\sum_{i=1}^{4} m_{i} g L_{s x}\right) d t
$$

\section{Conclusion}

According to the mathematical model, this article has conducted simulations in MATLAB, but the results cannot match well with the experiment results. Due to space limitations, results of simulations have to be omitted. Further studies to complete mathematical analysis works of the THR may include: 1) to consider effect of the constitutive model of SMA to the jumping property; 2) to seek optimal hop velocity and hop angle of a given hopping model.

\section{References}

[1] M.H. Kaplan, H. Seifert, Hopping transporters for lunar exploration, Journal of Spacecraft and Rockets, 6(1969)917-922.

[2] G.P. Sutton, M. Burrows, Biomechanics of jumping in the flea, Journal of Experimental Biology, 214(2011)836-847.

[3] M. Kovač, M. Fuchs, A. Guignard, J.C. Zufferey, et al, A miniature 7g jumping robot, IEEE International Conference on Robotics and Automation, (2008)373-378.

[4] M. Kovač, M. Schlegel, J.C. Zufferey, D. Floreano, Steerable miniature jumping robot, Auto Robot, 28(2010) 295-306.

[5] H.C. Bennet, E.C. A. Lucey, Jump of the flea: A study of the energetics and a model of the mechanism, Journal of Experimental Biology, 47(1967)59-76.

[6] http://www.st-andrews.ac.uk/ wjh/jumping/legwrk.htm.

[7] Y.Z. Liu, Dynamics about somersault swivel sports of human body, The Second Session of Sports Biomechanics Conference Proceedings, (1981)1-19.

[8] Y.Z. Liu, Qualitative theory of the jumping movement, Acta Mechanica Sinica, 26(1994)477-482.

[9] Y.Z. Liu, Swivel movement of a cat in a free falling, First Biomechanics Academic Conference, Beijing, (1981)388-393.

[10] Q. Zhang, A.J. Chen, Characteristics of the driving force of a bionic cricket microrobot in the take-off stage, Mechanical Science and Technology for Aerospace Engineering, 29(2010)621-624. 